Correspondence Alexandr Nemec anemec@szu.cz

\section{Acinetobacter bereziniae sp. nov. and Acinetobacter guillouiae sp. nov., to accommodate Acinetobacter genomic species 10 and 11, respectively}

\author{
Alexandr Nemec, ${ }^{1}$ Martin Musílek, ${ }^{2}$ Ondrej Šedo, ${ }^{3}$ Thierry De Baere, ${ }^{4}$ \\ Martina Maixnerová, ${ }^{1}$ Tanny J. K. van der Reijden, ${ }^{5}$ Zbyněk Zdráhal, ${ }^{3}$ \\ Mario Vaneechoutte ${ }^{4}$ and Lenie Dijkshoorn ${ }^{5}$ \\ ${ }^{1}$ Laboratory of Bacterial Genetics, National Institute of Public Health, Šrobárova 48, CZ-100 42 \\ Prague 10, Czech Republic \\ ${ }^{2}$ National Reference Laboratory for Meningococcal Infections, National Institute of Public Health, \\ Šrobárova 48, CZ-100 42 Prague 10, Czech Republic \\ ${ }^{3}$ Department of Functional Genomics and Proteomics, Institute of Experimental Biology, Faculty of \\ Science, Masaryk University, Kamenice 5 (Building A2), CZ-625 00 Brno, Czech Republic \\ ${ }^{4}$ Laboratory Bacteriology Research, Department of Clinical Chemistry, Microbiology and \\ Immunology, University of Ghent, 3 Blok A, De Pintelaan 185, B-9000 Gent, Belgium \\ ${ }^{5}$ Department of Infectious Diseases, Leiden University Medical Center C5-P, PO Box 9600, 2300 \\ RC Leiden, The Netherlands
}

\begin{abstract}
Acinetobacter genospecies (genomic species) 10 and 11 were described by Bouvet and Grimont in 1986 on the basis of DNA-DNA reassociation studies and comprehensive phenotypic analysis. In the present study, the names Acinetobacter bereziniae sp. nov. and Acinetobacter guillouiae sp. nov., respectively, are proposed for these genomic species based on the congruence of results of polyphasic analysis of 33 strains (16 and 17 strains of genomic species 10 and 11, respectively). All strains were investigated by selective restriction fragment amplification (i.e. AFLP) analysis $r p o B$ sequence analysis, amplified rDNA restriction analysis and tDNA intergenic length polymorphism analysis, and their nutritional and physiological properties were determined. Subsets of the strains were studied by $16 \mathrm{~S}$ rRNA gene sequence analysis and matrix-assisted laser desorption/ionization time-of-flight (MALDI-TOF) MS or had been classified previously by DNA-DNA reassociation. Results indicate that $A$. bereziniae and $A$. guillouiae represent two phenetically and phylogenetically distinct groups within the genus Acinetobacter. Based on the comparative analysis of housekeeping genes (16S rRNA and rpoB genes), these species together represent a monophyletic branch within the genus. Despite their overall phenotypic similarity, the ability to oxidize D-glucose and to grow at $38{ }^{\circ} \mathrm{C}$ can be used in the presumptive differentiation of these two species from each other: with the exception of three strains that were positive for only one test, $A$. bereziniae strains were positive for both tests, whereas $A$. guillouiae strains were negative in these tests. The strains of $A$. bereziniae originated mainly from human clinical specimens, whereas $A$. guillouiae strains were isolated from different environmental sources in addition to human specimens. The type strain of $A$. bereziniae sp. nov. is LMG $1003^{\top}\left(=\right.$ CIP $70.12^{\top}=$ ATCC $\left.17924^{\top}\right)$ and that of $A$. guillouiae sp. nov. is LMG $988^{\top}$ $\left(=\operatorname{CIP} 63.46^{\top}=\right.$ ATCC $11171^{\top}=$ CCUG $\left.2491^{\top}\right)$.
\end{abstract}

Abbreviations: ARDRA, amplified rDNA restriction analysis; MALDI-TOF MS, matrix-assisted laser desorption/ionization time-of-flight mass spectrometry; tDNA-PCR, tDNA-intergenic length polymorphism analysis.

The GenBank/EMBL/DDBJ accession numbers for the partial rpoB gene sequences determined in this study are FJ754429-FJ754463 and those for the 16S rRNA gene sequences of strains LUH 2634, LUH 9667, LUH 7013 and A23 are FM177773-FM177776, respectively.

Detailed data on the origins of strains of $A$. bereziniae and $A$. guillouiae spp. nov. and a dendrogram derived from cluster analysis of AFLP fingerprints are available as supplementary material with the online version of this paper. 
The genus Acinetobacter currently encompasses 19 species with validly published names and a number of tentative species with provisional designations (Dijkshoorn et al., 2007; Vaneechoutte \& De Baere, 2007; Vaneechoutte et al., 2009; Nemec et al., 2009). Acinetobacter genospecies 10 and 11 (here referred to as genomic species 10 and 11) were delineated in 1986 on the basis of DNA-DNA reassociation studies and comprehensive phenotypic analysis (Bouvet \& Grimont, 1986). As the number of strains in each group was small (four and three strains, respectively), the authors intentionally used provisional designations for these groups and did not propose formal species names. Later, other authors reported the isolation of strains belonging to genomic species 10 or 11 from various human, animal and environmental sources, which suggests that these genomic species are widespread biological entities (Tjernberg \& Ursing, 1989; Berlau et al., 1999; Chu et al., 1999; Nemec et al., 2000; Dijkshoorn et al., 2005). In this study, results of a polyphasic taxonomic analysis of strains of genomic species 10 and 11 are presented. Both our results and archive data published by others indicate that these genomic species represent two phenetically and phylogenetically distinct groups, which together represent a monophyletic branch within the genus. It is concluded that these groups represent two novel species for which the names Acinetobacter bereziniae sp. nov. and Acinetobacter guillouiae sp. nov., respectively, are proposed.

The 16 strains of genomic species 10 (A. bereziniae) and 17 strains of genomic species 11 (A. guillouiae) investigated in this study are listed in Table 1. Detailed information on the origin and properties of these strains is shown in Supplementary Table S1 (available in IJSEM Online).

Selective restriction fragment amplification (i.e. AFLP) analysis, a high-resolution DNA fingerprinting method, was used to assess overall genomic similarity of strains (Nemec et al., 2001; Dijkshoorn \& Nemec, 2007). Fingerprints of the strains were compared to a database of reference strains of all described named and unnamed Acinetobacter species. Results of comparative analysis of AFLP fingerprints of strains of A. bereziniae and A. guillouiae and of those representing all known Acinetobacter species are shown in Supplementary Fig. S1. The strains of A. bereziniae and $A$. guillouiae created two clusters at levels of 51 and $59 \%$, respectively, which are above the $50 \%$ level that has been established as the threshold for the delineation of Acinetobacter species (Nemec et al., 2001). The two clusters were separate from each other and from all other strains at similarity levels of $\leqslant 33 \%$. These data indicate both genomic coherence and distinctness of each of the two species.

Comparative sequence analysis of the RNA polymerase $\beta$-subunit $(r p o B)$ gene was performed as described previously (Nemec et al., 2009). Similarity calculations and cluster analysis were carried out for two concatenated zones encompassing nucleotide positions $2917-3267$ and 3322-3723. The rpoB-based dendrogram for strains of
A. bereziniae and A. guillouiae and for representative strains of other species of the genus is shown in Fig. 1. Intraspecies similarity values (expressed as percentages of identical nucleotides in corresponding positions in two aligned sequences) for strains of $A$. bereziniae and A. guillouiae were 98.9-100 and 97.6-100\%, respectively, whereas the similarity between these two species was $91.5-92.7 \%$. The interspecies similarity values of strains of $A$. bereziniae and A. guillouiae with other members of the genus (except for Acinetobacter baylyi CCM $7195^{\mathrm{T}}$ and C5; see below) were 78.5-86.6 and 77.6-87.5\%, respectively. When A. baylyi CCM $7195^{\mathrm{T}}$ and C5 were excluded from cluster analysis, strains of the two novel species formed two respective clusters each supported by a bootstrap value of $100 \%$; these clusters were linked to form a broader cluster, which was also supported by a bootstrap value of $100 \%$. These results demonstrate both the relatedness and distinctiveness of A. bereziniae and A. guillouiae and suggest that these species are monophyletic taxa that together represent a broader clade within the genus.

The $r p o B$ results obtained for the five A. baylyi strains deserve more detailed comment. Three of these strains (ADP1, A7 and 93A2) formed a relatively homogeneous cluster (intracluster similarity values of 99.3-99.8\%) that was markedly separate from other species of the genus (Fig. 1), with similarity values between the three A. baylyi strains and strains representing the other Acinetobacter species ranging from 78.5 to $83.9 \%$. In contrast, the two remaining A. baylyi strains (C5 and CCM $7195^{\mathrm{T}}$ ) clustered with the A. guillouiae strains and were only $82.2-88.1 \%$ similar to the other A. baylyi strains. However, although the $r p o B$ similarity between CCM $7195^{\mathrm{T}}$ and the $A$. guillouiae strains (97.6-99.7\%) was in the intraspecies range found for A. guillouiae, the similarity for A. baylyi $\mathrm{C} 5$ and the A. guillouiae strains was only $93.1-94.2 \%$. Comparative analysis of $r p o B$ sequences using nucleotide positions 2915-3775 revealed that the sequence of $A$. baylyi $\mathrm{C} 5$ consisted of two segments. Whereas the rpoB region of C5 between positions 2915 and 3134 corresponded to the rpoB orthologues of $A$. baylyi ADP1, A7 and 93A2 (similarity 99.1-100\%), the region between positions 3140 and 3775 corresponded to those of the A. guillouiae strains (similarity 98.5-99.7\%). These data suggest that the rpoB sequences of $A$. baylyi strains ADP1, A7 and 93A2 represent the authentic $A$. baylyi rpoB orthologues, whereas the two other A. baylyi strains (C5 and CCM $7195^{\mathrm{T}}$ ) carry sequences that may have resulted from intragenic recombination events following the acquisition of $r p o B$ sequences from A. guillouiae. This hypothesis is consistent with the fact that A. baylyi is known to be transformable by DNA from other Acinetobacter species (Juni, 1972; Vaneechoutte et al., 2006) and that both A. baylyi and A. guillouiae can be found in the same environment (Carr et al., 2003).

$16 \mathrm{~S}$ rRNA gene sequence analysis was carried out as described previously (Nemec et al., 2001; Vaneechoutte \& De Baere 2007). It included comparative analysis of $16 \mathrm{~S}$ rRNA gene sequences and amplified 16S rDNA restriction 
Table 1. Strains of Acinetobacter bereziniae sp. nov. and Acinetobacter guillouiae sp. nov.

Culture collections: ATCC, American Type Culture Collection, Manassas, USA; CCM, Czech Collection of Microorganisms, Brno, Czech Republic; CCUG, Culture Collection, University of Göteborg, Sweden; LMG, Bacteria Collection, Laboratorium voor Microbiologie Gent, Gent, Belgium; CIP, Collection de l'Institut Pasteur, Institut Pasteur, Paris, France. ANC and NIPH are strain designations used by A. Nemec and LUH and RUH are strain designations used by L. Dijkshoorn. CZ, Czech Republic; NL, Netherlands; in, inpatient; out, outpatient.

\begin{tabular}{|c|c|c|c|c|}
\hline Strain designation & Specimen & Location and year of isolation & Donor & Reference \\
\hline \multicolumn{5}{|l|}{ Acinetobacter bereziniae sp. nov. } \\
\hline $\begin{array}{c}\text { LMG } 1003^{\mathrm{T}}\left(=\text { NIPH } 521^{\mathrm{T}}=\text { ATCC }\right. \\
\left.17924^{\mathrm{T}}=\text { CIP } 70.12^{\mathrm{T}}=69^{\mathrm{T}}\right)\end{array}$ & Wound (human) & Before 1960 & & $\begin{array}{l}\text { Bouvet \& Grimont } \\
\text { (1986) }\end{array}$ \\
\hline CCUG $28268\left(=113: 2^{\star}\right)$ & Wound (human) & Malmö, Sweden, 1980 & I. Tjernberg & $\begin{array}{l}\text { Tjernberg \& Ursing } \\
\text { (1989) }\end{array}$ \\
\hline RUH $2222\left(=198^{\star}\right)$ & Urine (human) & Malmö, Sweden, 1980 & I. Tjernberg & $\begin{array}{l}\text { Tjernberg \& Ursing } \\
\text { (1989) }\end{array}$ \\
\hline NIPH 870 & Urine (human, in) & Č. Budějovice, CZ, 1997 & M. Horníková & Nemec et al. (2000) \\
\hline NIPH 1741 & Sputum (human, in) & Plzeň, CZ, 2001 & T. Bergerová & \\
\hline LUH 2634 & Sink (hospital environment) & Enschede, NL, 1995 & & \\
\hline LUH 6973 & Faeces (human, out) & Leiden, NL, 2000 & & \\
\hline LUH 9667 (=8630†) & Eye (rabbit) & Dublin, Ireland & S. Fanning & \\
\hline NIPH 3 & Hospital environment & Prague, 1991 & J. Vránková & \\
\hline NIPH 1050 & Urine (human, in) & Sedlčany, CZ, 1998 & P. Ježek & Nemec et al. (2000) \\
\hline NIPH 1054 & Faeces (human, out) & Př́bram, CZ, 1998 & P. Ježek & Nemec et al. (2000) \\
\hline LUH $5624(=\mathrm{R} 1-82 \dagger)$ & Sewage & Denmark, 1997 & L. Guardabassi & \\
\hline LUH 5852 & Sputum (human, in) & Leiden, NL, 1999 & & \\
\hline LUH $7438(=118 \mathrm{FFC} \dagger)$ & Blood (human) & Coimbra, Portugal, 1998 & G. da Silva & \\
\hline LUH 7832 (=V0112893†) & Wound (seal) & Utrecht, NL, 2001 & J. Wagenaar & \\
\hline LUH $8524(=130380-2 \dagger)$ & Clinical specimen (human) & Heerlen, NL, 2003 & Wagenvoort & \\
\hline \multicolumn{5}{|l|}{ Acinetobacter guillouiae sp. nov. } \\
\hline $\begin{array}{c}\text { LMG } 988^{\mathrm{T}}\left(=\text { NIPH } 522^{\mathrm{T}}=\text { ATCC }\right. \\
\left.11171^{\mathrm{T}}=\text { CIP } 63.46^{\mathrm{T}}=73^{\mathrm{T}}\right)\end{array}$ & Sewage & Before 1951 & & $\begin{array}{l}\text { Bouvet \& Grimont } \\
\text { (1986) }\end{array}$ \\
\hline RUH $2234\left(=174^{*}\right)$ & Contact lens & Malmö, Sweden, 1980 & I. Tjernberg & $\begin{array}{l}\text { Tjernberg \& Ursing } \\
\text { (1989) }\end{array}$ \\
\hline RUH $2236\left(=51^{\star}\right)$ & Urine (human) & Malmö, Sweden, 1980 & I. Tjernberg & $\begin{array}{l}\text { Tjernberg \& Ursing } \\
\text { (1989) }\end{array}$ \\
\hline NIPH 682 & Blood (human, in) & Č. Budějovice, CZ, 1997 & O. Hausner & Nemec et al. (2000) \\
\hline RUH $2860\left(=58 b^{*}\right)$ & Wound (human) & Malmö, Sweden, 1980 & I. Tjernberg & $\begin{array}{l}\text { Tjernberg \& Ursing } \\
\text { (1989) }\end{array}$ \\
\hline LUH $287\left(=225^{\star}\right)$ & Sputum (human) & Odense, Denmark, 1988-9 & P. Gerner-Smidt & $\begin{array}{l}\text { Gerner-Smidt et al. } \\
\text { (1991) }\end{array}$ \\
\hline RUH 1050 & Not known & Not known & J. van der Toorn & $\begin{array}{l}\text { Janssen et al. } \\
\text { (1997) }\end{array}$ \\
\hline $\mathrm{A}^{2} 3^{*}(=\mathrm{CIP} 107475)$ & Activated sludge & Albury, Australia & E. Carr & Carr et al. (2003) \\
\hline LUH $5606(=$ F4AUG7†) & Freshwater & Fish farm, Denmark, 1997 & L. Guardabassi & $\begin{array}{l}\text { Guardabassi et al. } \\
\text { (2000) }\end{array}$ \\
\hline LUH 5653 & Blood (human, in) & Leiden, NL, 1999 & & \\
\hline LUH 7830 (=V0112205†) & Eye (cat) & Utrecht, NL, 2001 & J. Wagenaar & \\
\hline LUH $4560(=\mathrm{A} 130 \mathrm{~d} \dagger)$ & Soil (barley field) & West Cornwall, UK, 1993-4 & H. Seifert & \\
\hline LUH 6980 & Faeces (human, out) & Leiden, NL, 2000 & & \\
\hline NIPH 991 & Ear swab (human, out) & Sedlčany, CZ, 1998 & P. Ježek & Nemec et al. (2000) \\
\hline ANC 3626 & Soil (beech forest) & CZ, 2007 & L. Kř́rížová & \\
\hline LUH 7013 & Faeces (human, out) & Leiden, NL, 2000 & & \\
\hline CCM 4725 & Raw milk & North Moravia, CZ & & \\
\hline
\end{tabular}

${ }^{*}$ Strain designation used in the reference publication.

$\dagger$ Strain designation used by the donor.

analysis (ARDRA), which is based on restriction endonuclease digestion of the amplified 16S rRNA gene (Dijkshoorn et al., 1998). In the present study, 16S rRNA gene sequences were obtained for $A$. bereziniae LUH 2634 and LUH 9667 and for A. guillouiae A23 and LUH 7013. The dendrogram of cluster analysis of these sequences and 


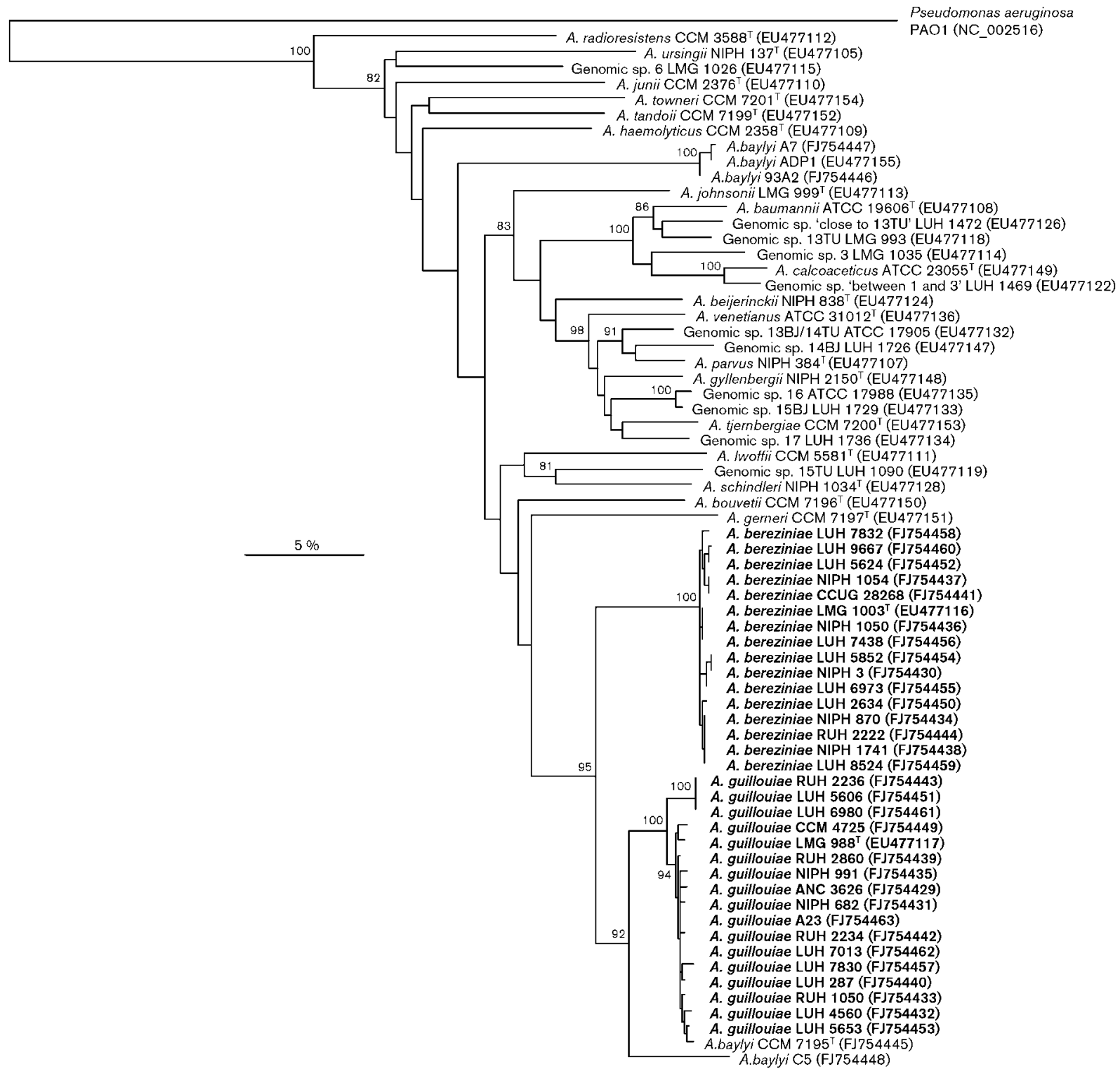

Fig. 1. Rooted neighbour-joining tree based on two concatenated zones of $r p o B$ of strains of $A$. bereziniae sp. nov. and $A$. guillouiae sp. nov. and representatives of all known species of the genus Acinetobacter. Cluster analysis was carried out using BioNumerics 5.1 (Applied Maths) with the $r p o B$ sequence of Pseudomonas aeruginosa PAO1 as the outgroup. Bootstrap values $\geqslant 80 \%$ (based on 1000 resamplings) are shown. GenBank accession numbers are given in parentheses. Bar, $5 \%$ sequence divergence.

the sequences of the type strains of $A$. bereziniae, $A$. guillouiae and other members of the genus Acinetobacter is shown in Fig. 2. The intraspecies sequence similarities of strains of $A$. bereziniae and A. guillouiae were 99.8-100 and $99.6-100 \%$, respectively. The interspecies sequence similarities between the strains of $A$. bereziniae and A. guillouiae were in the range 99.1-99.6\%, whereas the interspecies similarity between these strains and the type strains of other members of the genus Acinetobacter varied from $94.6 \%$ (Acinetobacter towneri) to $98.0 \%$ (Acinetobacter johnsonii). These data indicate a close re- latedness between A. bereziniae and A. guillouiae, as well as their distinctness from other species of the genus. The sequences of $A$. bereziniae and $A$. guillouiae formed two respective clusters in the $16 \mathrm{~S}$ rRNA gene-based dendrogram (Fig. 2), although they were supported only moderately by bootstrap values ( 86 and $69 \%$, respectively). The relative homogeneity of the $16 \mathrm{~S}$ rRNA gene sequences of the two species was also confirmed by ARDRA. Based on the ARDRA numbering system (Dijkshoorn et al., 1998), all $A$. bereziniae and $A$. guillouiae strains shared the patterns CfoI 4, AluI 2, MboI 1, RsaI 2 and MspI 3 and only differed in 


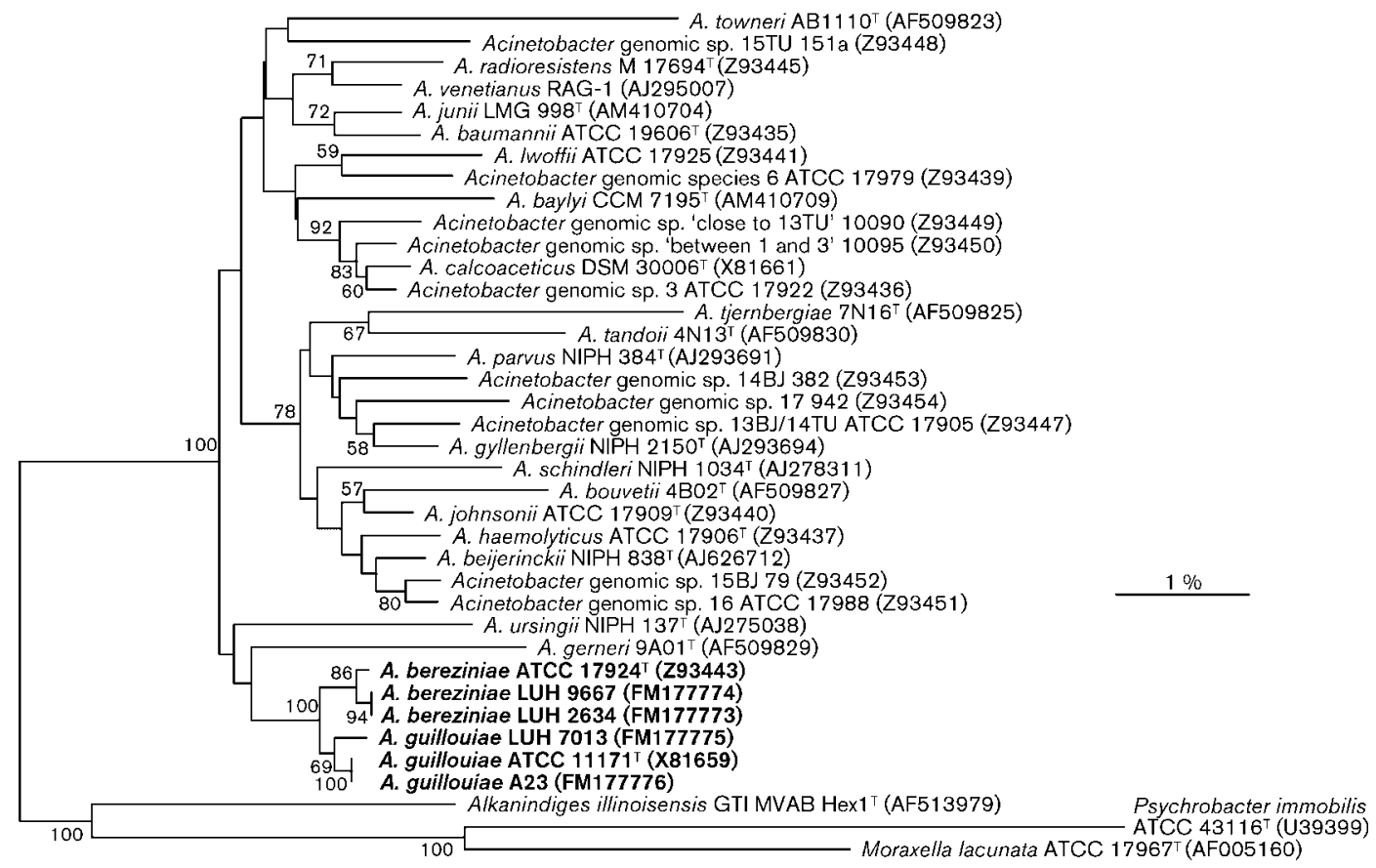

Fig. 2. Rooted $16 \mathrm{~S}$ rRNA gene sequence-based tree showing the relationships between strains of $A$. bereziniae sp. nov. and A. guillouiae sp. nov., other members of the genus Acinetobacter and representatives of closely related genera. The cluster analysis, performed using GENEBASE (Applied Maths), was based on the neighbour-joining method using Alkanindiges illinoisensis GTI MVAB Hex1 ${ }^{\top}$, Psychrobacter immobilis ATCC $43116^{\top}$ and Moraxella lacunata ATCC $17967^{\top}$ as the outgroup, an open gap penalty of $100 \%$ and a unit gap cost of $20 \%$. The region used corresponds to Escherichia coli positions $99-1470$. Bootstrap percentages ( $\geqslant 50 \%)$ after 100 resamplings are shown. GenBank/EMBL accession numbers are given in parentheses. Bar, $1 \%$ sequence divergence.

the BsmaI pattern: strains of A. bereziniae yielded BsmaI pattern 1, whereas those of A. guillouiae had BsmaI pattern 2.

DNA-DNA reassociation studies to delineate genomic species 10 and 11 have been performed previously in the studies of Bouvet \& Grimont (1986) and Tjernberg \& Ursing (1989). In the former study, four and three strains of genomic species 10 and 11, respectively, were investigated by DNA-DNA reassociation. The four strains of genomic species 10 , including $A$. bereziniae LMG $1003^{\mathrm{T}}$ $\left(=69^{\mathrm{T}}\right)$, were $65-68 \%$ similar to each other, with $\Delta T_{\mathrm{m}}$ ranging from 0.8 to $1.2{ }^{\circ} \mathrm{C}$, whereas the three strains of genomic species 11, including A. guillouiae LMG $988^{\mathrm{T}}$ $\left(=73^{\mathrm{T}}\right)$, were $71-74 \%$ similar to one another. In contrast, genomic species 10 and 11 were only $21-30 \%$ similar to each other and the DNA-DNA reassociation values between these species and the strains of the other 10 genomic species were in the range 3-21\%. Similar results have been obtained by Tjernberg \& Ursing (1989). In their study, three strains, including A. bereziniae LMG $1003^{\mathrm{T}}$ $\left(=\right.$ ATCC $\left.17924^{\mathrm{T}}\right)$, RUH $2222(=198)$ and CCUG 28268 $(=113: 2)$, were allocated to genomic species 10 , whereas five strains, including LMG $988^{\mathrm{T}}\left(=\mathrm{CIP} 63.46^{\mathrm{T}}\right)$, RUH $2234(=174)$, RUH $2236(=51)$ and RUH $2860(=58 \mathrm{~b})$, were classified as genomic species 11 . These archive data further support the distinctiveness of the genomes of
A. bereziniae and A. guillouiae from each other and from the other (genomic) species described by Bouvet \& Grimont (1986) and Tjernberg \& Ursing (1989).

tDNA-intergenic length polymorphism analysis (tDNAPCR) was carried out using one fluorescent primer to allow visualization of the amplified intergenic tRNA-spacer regions using ABI 310 capillary electrophoresis apparatus as described previously (Vaneechoutte et al., 1998; Baele et al., 2000, 2001). Electrophoretograms were compared with BaseHopper, a software program developed at Ghent University (Baele et al., 2001), available upon request from the authors. Both species yielded more than 50 amplified tRNA-intergenic spacer fragments, ranging between 50 and $477 \mathrm{bp}$ in length. Most fragments were observed for both species, indicating their relatedness, but several fragments enabled differentiation between them: spacers with lengths of $139.6 \pm 0.3$ (mean $\pm \mathrm{SD}), 166.3 \pm 0.2,181.6 \pm 0.2,220.7 \pm 0.1$, $228.6 \pm 0.2$ and $238.5 \pm 0.3$ bp were observed exclusively for A. bereziniae and present in almost all strains of this species, whereas spacers with lengths of $109.6 \pm 0.4,173.6 \pm 0.3$, $221.7 \pm 0.1,229.7 \pm 0.1,235.6 \pm 0.5$ and $477.7 \pm 1.2$ bp were present exclusively in almost all A. guillouiae strains.

Results of phenotypic tests are given in Table 2 and in the species descriptions. All tests have been described in detail 
by Nemec et al. (2009). Strains of A. bereziniae and A. guillouiae were unable to lyse sheep erythrocytes in the blood agar test or produce gelatinase, but they were able to grow on a number of carbon sources. Table 2 shows phenotypic characters that are useful for the differentiation of these species from the Acinetobacter species that include non-haemolytic and/or non-proteolytic strains. A unique property of both A. bereziniae and A. guillouiae compared to all known Acinetobacter species was the ability to grow on histamine, although only $63-65 \%$ of strains of these species were positive in this test. The two species showed an overall phenotypic similarity to each other, leaving only a few tests that could be used for their presumptive differentiation from each other. With three exceptions, the $A$. bereziniae strains oxidized $\mathrm{D}$-glucose and grew in brain-heart infusion (BHI) agar at $38{ }^{\circ} \mathrm{C}$, whereas A. guillouiae strains were negative in these tests. The exceptions included two A. bereziniae strains (NIPH 3 and LUH 9667) that were negative in the D-glucose oxidation test and one A. guillouiae strain (NIPH 682) that could grow at $38{ }^{\circ} \mathrm{C}$. In addition, $82 \%$ of the A. guillouiae strains grew on tryptamine, whereas none of the A. bereziniae strains utilized this carbon source. However, growth of A. guillouiae strains on tryptamine was usually slow and visible only after 4 or 6 days of incubation.

Matrix-assisted laser desorption/ionization time-of-flight (MALDI-TOF) MS measurements were carried out using

Table 2. Differential phenotypic properties of $A$. bereziniae sp. nov., $A$. guillouiae sp. nov. and other species comprising nonhaemolytic or proteolytic strains

Species: 1, A. bereziniae sp. nov. (16 strains); 2, A. guillouiae sp. nov. (17); 3, A. baumannii (8); 4, A. calcoaceticus (3); 5, genomic species 3 (7); 6, genomic species $13 \mathrm{TU}$ (9); 7, A. junii (4); 8, A. johnsonii (8); 9, A. lwoffii (8); 10, A. radioresistens (6); 11, A. ursingii (15); 12, A. schindleri (13); 13, A. parvus (10); 14, A. baylyi (5); 15, A. towneri (2); 16, A. bouvetii (1); 17, A. gerneri (1); 18, A. tandoii (1). All results were obtained in the present study. Type or reference strains $(n=103)$ of previously described genomic species were selected from the studies of Bouvet \& Grimont (1986), Tjernberg \& Ursing (1989), Janssen et al. (1997), Carr et al. (2003), Nemec et al. (2001, 2003) and Vaneechoutte et al. (2009). +, All strains positive within 4 days of incubation; - all strains negative after 10 days of incubation; $v+, 85-99 \%$ of strains positive within 6 days of incubation; v, 16$84 \%$ of strains positive within 6 days of incubation; $v-, 1-15 \%$ of strains positive within 6 days of incubation; ND, not determined. For A. bereziniae and A. guillouiae, percentages of positive reactions are indicated for strain-dependent reactions.

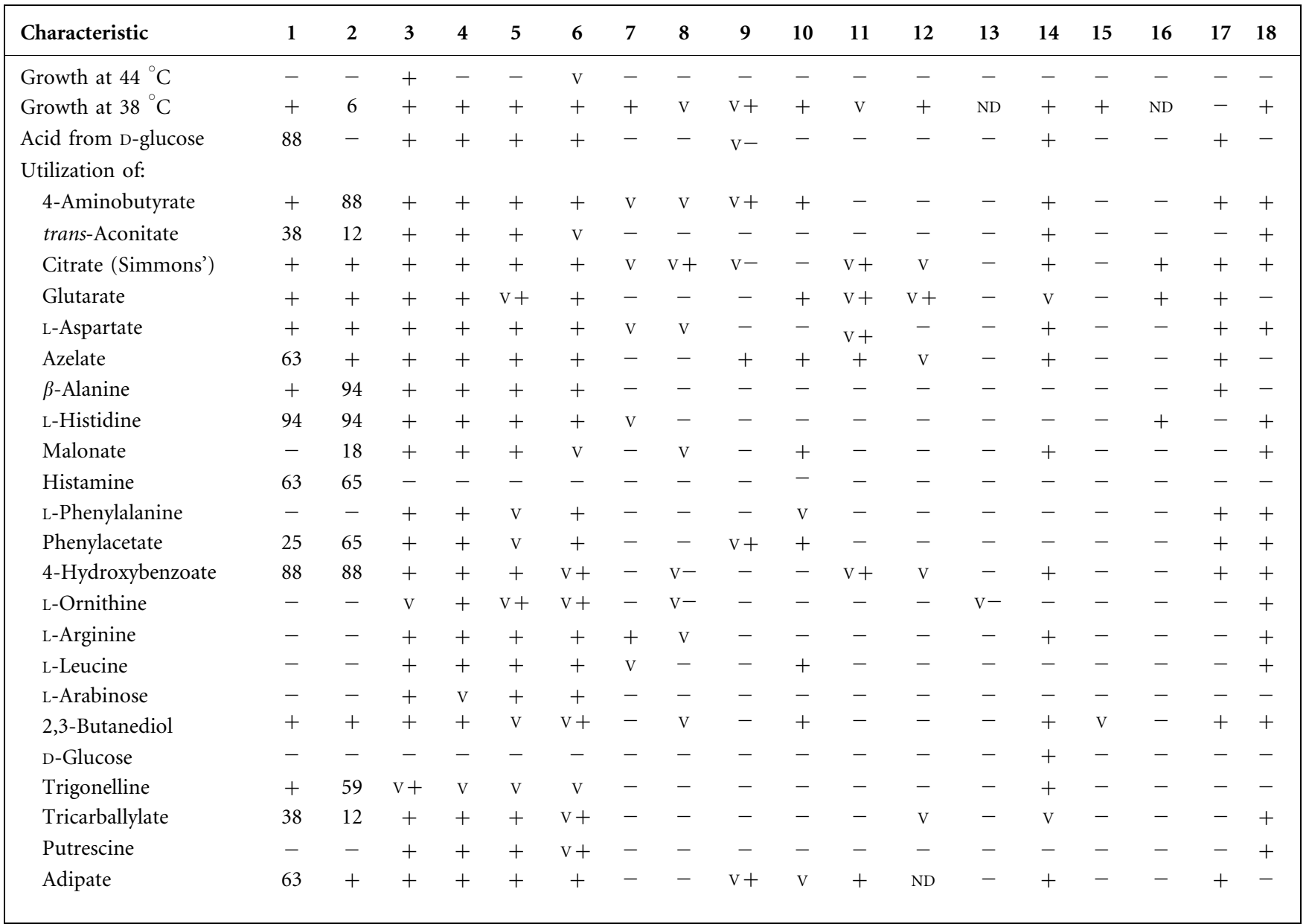


an Ultraflex III instrument (Bruker Daltonik) operated in linear positive mode. External calibration of the mass spectra was performed using Escherichia coli DH5 $\alpha$ standard peaks. Preparation of samples included sterilization in $75 \%$ ethanol and protein extraction with an acetonitrile/formic acid/water $(50: 35: 15)$ mixture. Aliquots of protein extracts $(0.3 \mu \mathrm{l}$ per well $)$ were deposited on the MALDI target and overlaid with $0.3 \mu \mathrm{l}$ saturated solution of an $\alpha$-cyano- 4 hydroxycinnamic acid in acetonitrile/water/trifluoroacetic acid $(50: 47.5: 2.5)$ mixture (Williams et al., 2003). Each extract was spotted onto three MALDI target wells. Five consecutive spectrum accumulations including 1000 laser shots aimed using a lattice raster were acquired from each of the wells. Obtained spectra were processed using BioTyper software (version 1.1; Bruker Daltonik). Signals present in at least $70 \%$ of the spectrum accumulations were taken into account for statistical analysis. The MALDI-TOF MS-based dendrogram was generated using the correlation distance measure with the mean linkage algorithm. MALDI-TOF MS analysis of 11 strains of each of A. bereziniae and A. guillouiae showed specific signals for all representatives of the particular species, namely peaks 7156, 7407 and $7796(\mathrm{~m} / \mathrm{z})$ for A. bereziniae and 3258, 3690, 6513, 6978, 7378 and $7813(\mathrm{~m} / \mathrm{z})$ for A. guillouiae (see Supplementary Fig. S2). Supplementary Fig. S2 also shows the results of cluster analysis, which demonstrate a clear separation of the respective mass spectra of A. bereziniae and A. guillouiae.

\section{Description of Acinetobacter bereziniae sp. nov.}

Acinetobacter bereziniae (be.re.zi.ni'ae. N.L. fem. gen. n. bereziniae of Bérézin, named after Eugénie BergogneBérézin, French medical microbiologist).

Phenotypic characteristics correspond to those of the genus (Baumann et al., 1968), i.e. Gram-stain-negative, strictly aerobic, oxidase-negative, catalase-positive, non-motile coccobacillus, capable of growing in mineral media with acetate as sole carbon source and ammonia as sole source of nitrogen, and incapable of dissimilative denitrification. Positive in the transformation assay of Juni (1972). The species description is based on characterization of 16 strains. Colonies on tryptic soy agar (TSA; Oxoid) after $24 \mathrm{~h}$ incubation at $30{ }^{\circ} \mathrm{C}$ are $1.0-2.0 \mathrm{~mm}$ in diameter, circular, convex, smooth and slightly opaque with entire margins. Growth occurs in BHI (Oxoid) at $25-38{ }^{\circ} \mathrm{C}$, but not at $41{ }^{\circ} \mathrm{C}$. Acid is produced from D-glucose by the majority of strains ( $88 \%$ of the strains studied), gelatin is not hydrolysed and haemolysis is not observed on agar media supplemented with sheep erythrocytes. Acetate, ethanol, DL-lactate, 4-aminobutyrate, citrate (Simmons'), glutarate, L-aspartate, $\beta$-alanine, 2,3-butanediol, trigonelline, benzoate and L-glutamate are utilized as sole sources of carbon with growth visible within 4 days (generally 2 days) of incubation. No growth on malonate, L-phenylalanine, laevulinate, citraconate, L-tartrate, L-ornithine, L-arginine, L-leucine, D-glucose, D-gluconate, D-ribose, L-arabinose, gentisate, tryptamine or putrescine occurs within 10 days.
Some strains utilize trans-aconitate $(38 \%$ of the strains studied), azelate (63\%), L-histidine (94\%), D-malate ( $88 \%$ ), histamine (63\%), phenylacetate ( $25 \%)$, 4-hydroxybenzoate $(88 \%)$, adipate $(63 \%)$ and tricarballylate $(38 \%)$ within 6 days.

The type strain is LMG $1003^{\mathrm{T}}\left(=\mathrm{CIP} 70.12^{\mathrm{T}}=\mathrm{ATCC}\right.$ $17924^{\mathrm{T}}$ ), isolated from a human wound before 1960. It was used as the reference strain of genospecies 10 by Bouvet \& Grimont (1986). This strain produces acid from D-glucose and grows on azelate, L-histidine, D-malate, histamine, 4-hydroxybenzoate and adipate, but does not utilize transaconitate, phenylacetate or tricarballylate. Strain LMG $1003^{\mathrm{T}}$ produces two stable colony types.

\section{Description of Acinetobacter guillouiae sp. nov.}

Acinetobacter guillouiae (guil.lou'i.ae. N.L. fem. gen. n. guillouiae of Guillou, named after Marie-Laure JolyGuillou, French medical microbiologist).

Phenotypic characteristics correspond to those of the genus (Baumann et al., 1968), i.e. Gram-stain-negative, strictly aerobic, oxidase-negative, catalase-positive, non-motile coccobacillus, capable of growing in mineral media with acetate as sole carbon source and ammonia as sole source of nitrogen, and incapable of dissimilative denitrification. Positive in the transformation assay of Juni (1972). The species description is based on characterization of 17 strains. Colonies on TSA after $24 \mathrm{~h}$ incubation at $30{ }^{\circ} \mathrm{C}$ are $1.0-2.0 \mathrm{~mm}$ in diameter, circular, convex, smooth and slightly opaque with entire margins. Growth occurs in BHI at $25-35{ }^{\circ} \mathrm{C}$; no growth usually occurs at $38{ }^{\circ} \mathrm{C}$. Acid is not produced from D-glucose, gelatin is not hydrolysed and haemolysis is not observed on agar media supplemented with sheep erythrocytes. Acetate, ethanol, DL-lactate, azelate, citrate (Simmons'), glutarate, L-aspartate, 2,3-butanediol, adipate and L-glutamate are utilized as sole sources of carbon with growth visible within 4 days (generally 2 days) of incubation. No growth on L-phenylalanine, laevulinate, citraconate, L-tartrate, L-ornithine, L-arginine, L-leucine, D-glucose, D-gluconate, D-ribose, L-arabinose or putrescine occurs within 10 days. Some strains utilize trans-aconitate ( $12 \%$ of the strains studied), 4-aminobutyrate $(88 \%)$, $\beta$-alanine (94\%), L-histidine (94\%), D-malate (94\%), histamine $(65 \%)$, phenylacetate $(65 \%)$, 4-hydroxybenzoate $(88 \%)$, malonate $(18 \%)$, gentisate $(18 \%)$, trigonelline $(59 \%)$, tryptamine $(82 \%)$, benzoate $(88 \%)$ and tricarballylate $(12 \%)$ within 6 days.

The type strain is LMG $988^{\mathrm{T}}\left(=\mathrm{CIP} \quad 63.46^{\mathrm{T}}=\mathrm{ATCC}\right.$ $11171^{\mathrm{T}}=$ CCUG $2491^{\mathrm{T}}$ ), isolated from sewage containing gas-work effluent before 1951. It was used as the reference strain of genospecies 11 by Bouvet \& Grimont (1986). This strain grows on $\beta$-alanine, L-histidine, D-malate, histamine, phenylacetate, 4-hydroxybenzoate, tryptamine and benzoate, but does not grow at $38{ }^{\circ} \mathrm{C}$ or utilize trans-aconitate, malonate, gentisate, trigonelline or tricarballylate. Growth of the type strain on 4-aminobutyrate is not reproducible. 


\section{Acknowledgements}

We thank all the donors listed in Table 1 for generous provision of strains. A.N. was supported by grant no. MSM0021620812 and Z.Z. and O. S. were supported by grant no. MSM0021622415 of the Czech Ministry of Education, Youth and Sports. T. D. B. is indebted to the Fund for Scientific Research-Flanders (FWO) for a postdoctoral fellowship.

\section{References}

Baele, M., Baele, P., Vaneechoutte, M., Storms, V., Butaye, P., Devriese, L. A., Verschraegen, G., Gillis, M. \& Haesebrouck, F. (2000). Application of tRNA intergenic spacer PCR for identification of Enterococcus species. J Clin Microbiol 38, 4201-4207.

Baele, M., Storms, V., Haesebrouck, F., Devriese, L. A., Gillis, M., Verschraegen, G., de Baere, T. \& Vaneechoutte, M. (2001). Application and evaluation of the interlaboratory reproducibility of tRNA intergenic length polymorphism analysis (tDNA-PCR) for identification of Streptococcus species. J Clin Microbiol 39, 1436-1442.

Baumann, P., Doudoroff, M. \& Stanier, R. Y. (1968). A study of the Moraxella group. II. Oxidative-negative species (genus Acinetobacter). J Bacteriol 95, 1520-1541.

Berlau, J., Aucken, H. M., Houang, E. \& Pitt, T. L. (1999). Isolation of Acinetobacter spp. including A. baumannii from vegetables: implications for hospital-acquired infections. J Hosp Infect 42, 201-204.

Bouvet, P. J. M. \& Grimont, P. A. D. (1986). Taxonomy of the genus Acinetobacter with the recognition of Acinetobacter baumannii sp. nov., Acinetobacter haemolyticus sp. nov., Acinetobacter johnsonii sp. nov., and Acinetobacter junii sp. nov. and emended descriptions of Acinetobacter calcoaceticus and Acinetobacter lwoffii. Int J Syst Bacteriol 36, 228-240.

Carr, E. L., Kämpfer, P., Patel, B. K. C., Gürtler, V. \& Seviour, R. J. (2003). Seven novel species of Acinetobacter isolated from activated sludge. Int J Syst Evol Microbiol 53, 953-963.

Chu, Y. W., Leung, C. M., Houang, E. T., Ng, K. C., Leung, C. B., Leung, H. Y. \& Cheng, A. F. (1999). Skin carriage of acinetobacters in Hong Kong. J Clin Microbiol 37, 2962-2967.

Dijkshoorn, L. \& Nemec, A. (2007). The diversity of the genus Acinetobacter. In Acinetobacter Molecular Biology, pp. 1-34. Edited by U. Gerischer. Wymondham, UK: Horizon Scientific Press/Caister Academic Press.

Dijkshoorn, L., van Harsselaar, B., Tjernberg, I., Bouvet, P. J. M. \& Vaneechoutte, M. (1998). Evaluation of amplified ribosomal DNA restriction analysis for identification of Acinetobacter genomic species. Syst Appl Microbiol 21, 33-39.

Dijkshoorn, L., van Aken, E., Shunburne, L., van der Reijden, T. J., Bernards, A. T., Nemec, A. \& Towner, K. J. (2005). Prevalence of Acinetobacter baumannii and other Acinetobacter spp. in faecal samples from non-hospitalised individuals. Clin Microbiol Infect 11, 329-332.

Dijkshoorn, L., Nemec, A. \& Seifert, H. (2007). An increasing threat in the hospital: multidrug resistant Acinetobacter baumannii. Nat Rev Microbiol 5, 939-951.
Gerner-Smidt, P., Tjernberg, I. \& Ursing, J. (1991). Reliability of phenotypic tests for identification of Acinetobacter species. J Clin Microbiol 29, 277-282.

Guardabassi, L., Dijkshoorn, L., Collard, J. M., Olsen, J. E. \& Dalsgaard, A. (2000). Distribution and in-vitro transfer of tetracycline resistance determinants in clinical and aquatic Acinetobacter strains. J Med Microbiol 49, 929-936.

Janssen, P., Maquelin, K., Coopman, R., Tjernberg, I., Bouvet, P., Kersters, K. \& Dijkshoorn, L. (1997). Discrimination of Acinetobacter genomic species by AFLP fingerprinting. Int J Syst Bacteriol 47, 11791187.

Juni, E. (1972). Interspecies transformation of Acinetobacter: genetic evidence for a ubiquitous genus. J Bacteriol 112, 917-931.

Nemec, A., Dijkshoorn, L. \& Ježek, P. (2000). Recognition of two novel phenons of the genus Acinetobacter among non-glucoseacidifying isolates from human specimens. J Clin Microbiol 38, 3937-3941.

Nemec, A., De Baere, T., Tjernberg, I., Vaneechoutte, M., van der Reijden, T. J. K. \& Dijkshoorn, L. (2001). Acinetobacter ursingii sp. nov. and Acinetobacter schindleri sp. nov., isolated from human clinical specimens. Int J Syst Evol Microbiol 51, 1891-1899.

Nemec, A., Dijkshoorn, L., Cleenwerck, I., De Baere, T., Janssens, D., van der Reijden, T. J. K., Ježek, P. \& Vaneechoutte, M. (2003). Acinetobacter parvus sp. nov., a small-colony-forming species isolated from human clinical specimens. Int J Syst Evol Microbiol 53, 15631567.

Nemec, A., Musilek, M., Maixnerová, M., De Baere, T., van der Reijden, T. J. K., Vaneechoutte, M. \& Dijkshoorn, L. (2009). Acinetobacter beijerinckii sp. nov. and Acinetobacter gyllenbergii sp. nov., haemolytic organisms isolated from humans. Int J Syst Evol Microbiol 59, 118-124.

Tjernberg, I. \& Ursing, J. (1989). Clinical strains of Acinetobacter classified by DNA-DNA hybridization. APMIS 97, 595-605.

Vaneechoutte, M. \& De Baere, T. (2007). Taxonomy of the genus Acinetobacter, based on $16 \mathrm{~S}$ ribosomal RNA gene sequences. In Acinetobacter Molecular Biology, pp. 35-60. Edited by U. Gerischer. Wymondham, UK: Horizon Scientific Press/Caister Academic Press.

Vaneechoutte, M., Boerlin, P., Tichy, H.-V., Bannerman, E., Jäger, B. \& Bille, J. (1998). Comparison of PCR-based DNA fingerprinting techniques for the identification of Listeria species and their use for atypical Listeria isolates. Int J Syst Bacteriol 48, 127-139.

Vaneechoutte, M., Young, D. M., Ornston, L. N., De Baere, T., Nemec, A., van der Reijden, T., Carr, E., Tjernberg, I. \& Dijkshoorn, L. (2006). Naturally transformable Acinetobacter sp. strain ADP1 belongs to the newly described species Acinetobacter baylyi. Appl Environ Microbiol 72, 932-936.

Vaneechoutte, M., Nemec, A., Musilek, M., van der Reijden, T. J. K., van den Barselaar, M., Tjernberg, I., Calame, W., Fani, R., De Baere, T. \& Dijkshoorn, L. (2009). Description of Acinetobacter venetianus ex Di Cello et al. 1997 sp. nov. Int J Syst Evol Microbiol 59, 1376-1381.

Williams, T. L., Andrzejewski, D., Lay, J. O. \& Musser, S. M. (2003). Experimental factors affecting the duality and reproducibility of MALDI TOF mass spectra obtained from whole bacteria cells. J Am Soc Mass Spectrom 14, 342-351. 